\title{
A note on the tangent bundle
}

\author{
Mohammad Nazrul Islam Khan \\ Department of Computer Engineering, College of Computer, Qassim University, Al-Qassim, Saudi Arabia
}

Received: 8 January 2017, Accepted: 2 November 2017

Published online: 29 January 2018.

\begin{abstract}
The aim of the paper is to study Lorentzian almost para contact structure in the tangent bundle. Some results related to Lie derivative is investigated.
\end{abstract}

Keywords: Tangent bundle, vertical lift, complete lift and horizontal lift, Lie derivative.

\section{Introduction}

The differential geometry of tangent bundles has valuable vicinity in the differential geometry because it provides numerous innovative problems in the study of modern differential geometry. Numerous investigators made valuable contributions on differential geometry of tangent bundles including Yano and Ishihara [8], Davies [9], Yano and Davies [10]. The complete, vertical and horizontal lifts of tensor fields and connections on any manifold $\mathrm{M}$ to tangent manifold TM has been studied by Yano and Ishihara [3]. Furthermore, Das and the author [2] have obtained almost product structure by means of the complete, vertical and horizontal lifts of almost r-contact structures on tangent bundles. Tekkoyun [6] produced almost para-complex structures on tangent bundle by using lifting theory. The second author [4, 7] has studied lifts of hypersurface with connections to tangent bundles and Kähler manifold. In the present work, we recall tangent bundle, complete lift, vertical lift, almost product structure and almost product structure in section 2 . The section 3 is devoted to study complete lift of Lorentzian almost para contact structure in the tangent bundle. In the last section, the horizontal lift of the structure in tangent bundle is also studied.

\section{Preliminaries}

Let $M$ be an n-dimensional differentiable manifold of class $C^{\infty}$ and $T_{p}(M)$ be the tangent space of $M$ at a point of $M$. Then the set

$$
T(M)=\bigcup_{p \in M} T_{p}(M)
$$

is called the tangent bundle over the manifold $M[4]$.

\subsection{Vertical lifts}

If $f$ is a function in $M$, we write $f^{V}$ for the function in $T(M)$ obtained by forming the composition of $\pi: T(M \longrightarrow) M$ and $f: M \longrightarrow R$, so that

$$
f^{V}=f o \pi
$$


thus, if a point $\tilde{p} \varepsilon \pi^{-1}(U)$ has induced coordinates $\left(x^{h}, y^{h}\right)$ then

$$
f^{V}(\tilde{p})=f^{V}(x, y)=f o \pi(\tilde{p})=f(p)=f(x)
$$

thus the value of $f^{V}(\tilde{p})$ is constant along each fibre $T_{p}(M)$ and equal to the value $f(p)$. We call $f^{V}$ the vertical lift of the function $f$. Vertical lifts to a unique algebraic isomorphism of the tensor algebra $\mathfrak{I}(M)$ into the tensor algebra $\mathfrak{I}(T(M))$ with respect to constant coefficients by the conditions

$$
(P \otimes Q)^{V}=P^{V} \otimes Q^{V},(P+R)^{V}=P^{V}+R^{V}
$$

$P, Q$ and $R$ being arbitrary elements of $\mathfrak{I}(M)[4]$.

\subsection{Complete lifts}

If $f$ is a function in $M$, we write $f^{C}$ for the function in $T(M)$ defined by

$$
f^{C}=i(d f)
$$

and call $f^{C}$ the complete lift of the function $f$. The complete lift $f^{C}$ of a function $f$ has the local expression

$$
f^{C}=y^{i} \partial_{i} f=\partial f
$$

with respect to the induced coordinates in $T(M)$, where $\partial f$ denotes $y^{i} \partial_{i} f$. Suppose that $X \in \mathfrak{I}_{0}^{1}(M)$. We define a vector field $X^{C}$ in $T(M)$ by

$$
X^{C} f^{C}=(X f)^{C}
$$

$f$ being an arbitrary function in $M$ and call $X^{C}$ the complete lift of $X$ in $T(M)$. The complete lift $X^{C}$ of $X$ with components $x^{h}$ in $M$ has components

$$
X^{C}:\left[\begin{array}{r}
x^{h} \\
\partial x^{h}
\end{array}\right]
$$

with respect to the induced coordinates in $T(M)$.

Suppose that $X \in \mathfrak{I}_{0}^{1}(M)$ Then a 1 -form $\omega^{C}$ in $T(M)$ defined by

$$
\omega^{C}\left(X^{C}\right)=(\omega(X))^{C}
$$

$X$ being an arbitrary vector field in $M$. We call $\omega^{C}$ the complete lift of $\omega$.

The complete lifts to a unique algebra isomorphism of the tensor algebra $J(M)$ into the tensor algebra $\mathfrak{I}(T(M))$ with respect to constant coefficients, is given by the conditions

$$
(P \otimes Q)^{C}=P^{C} \otimes Q^{V}+P^{V} \otimes Q^{C},(P+R)^{C}=P^{C}+R^{C}
$$

$P, Q$ and $R$ being arbitrary elements of $\mathfrak{I}(M)$. 


\subsection{Horizontal lifts}

The horizontal lift $f^{H}$ of $f \in \mathfrak{I}_{0}^{0}(M)$ to the tangent bundle $T(M)$ by

$$
f^{H}=f^{C}-\nabla_{\gamma} f
$$

where $\nabla_{\gamma} f=\gamma(\nabla f)$. Let $X \in \mathfrak{I}_{0}^{1}(M)$. Then the horizontal lift $X^{H}$ of $\mathrm{X}$ defined by

$$
X^{H}=X^{C}-\nabla_{\gamma} X
$$

in $\mathrm{T}(\mathrm{M})$, where $\nabla_{\gamma} X=\gamma(\nabla X)$. The horizontal lift $X^{H}$ of $X$ has the components

$$
X^{H}:\left[\begin{array}{r}
x^{h} \\
-\Gamma_{i}^{h} x^{i}
\end{array}\right]
$$

with respect to the induced coordinates in $T(M)$, where

$$
\Gamma_{i}^{h}=y^{j} \Gamma_{j}^{h} i
$$

The horizontal lift $S^{H}$ of $X$ a tensor field $S$ of arbitrary type in $M$ to $T(M)$ is defined by

$$
S^{H}=S^{C}-\nabla_{\gamma} S
$$

For any $P, Q \in \mathfrak{I}(M)$. We have

$$
\nabla_{\gamma}(P \otimes Q)=\left(\nabla_{\gamma} P\right) \otimes Q^{V}+P^{V} \otimes\left(\nabla_{\gamma} Q\right)
$$

or

$$
(P \otimes Q)^{H}=P^{H} \otimes Q^{V}+P^{V} \otimes Q^{V}
$$

\subsection{Almost Product Structure}

Let $M^{n}$ be an n-dimensional differentiable manifold of class $C^{\infty}$. If there exists on $C^{\infty}$, a tensor field $F$ of type $(1,1)$ and of class $C^{\infty}$ such that

$$
F^{2}=I
$$

where $I$ denotes the unit tensor field, we say that $\mathrm{F}$ gives to an almost product structure [2].

\section{Complete lifts of almost product structure and Lorentzian almost para contact structure in the tangent bundle}

Let $\bar{M}$ be an $2 n+1$-dimensional differentiable manifold of class $C^{\infty}$ and $T(\bar{M})$ denotes the tangent bundle of $\bar{M}$. Suppose there is given in $\bar{M}$, a tensor field $F(1,1)$, vector field $U$ and 1-forms $\omega$ satisfying

$$
F^{2}=I+U \otimes \omega
$$


where
(i) $\quad F U=0$
(ii) $\omega \circ F=0$
(iii) $\omega(U)=1$

thus the manifold $\bar{M}$ satisfying conditions (1), (2) will be said to possess Lorentzian almost para contact structure [1].

Theorem 1. Let $\bar{M}$ be a differentiable Manifold endowed with Lorentzian almost para contact structure $(F, U, \omega)$. Prove that

$$
\tilde{J}=F^{C}+\left(U^{V} \otimes \omega^{V}-U^{C} \otimes \omega^{C}\right.
$$

is almost product structure on $T(\bar{M})$.

Proof. From (1) and (2), we have

$$
\left(F^{C}\right)^{2}=I+\left(U^{V} \otimes \omega^{C}-U^{C} \otimes \omega^{V}\right)
$$

and

(i) $F^{C} U^{V}=0, \quad F^{C} U^{C}=0$

(ii) $\omega^{V} \circ F^{C}=0, \omega^{C} \circ F^{V}=0, \omega^{C} \circ F^{C}=0$,

(iii) $\omega^{V}\left(U^{V}\right)=0, \omega^{V}\left(U^{C}\right)=1, \omega^{C}\left(U^{V}\right)=1, \omega^{C}\left(U^{C}\right)=0$.

Let us define an element $\tilde{J}$ of $J(T(\bar{M}))$ by

$$
\tilde{J}=F^{C}+\left(U^{V} \otimes \omega^{V}-U^{C} \otimes \omega^{C}\right.
$$

then we find by (3), (4) and (5),

$$
\tilde{J}^{2}=I
$$

thus $\tilde{J}$ is an almost product structure in $T(\bar{M})$.

In view of equation (5), we have

$$
\begin{gathered}
\tilde{J} X^{V}=-(F X)^{V}+(\omega(X))^{V} U^{C} \\
\tilde{J} X^{C}=-(F X)^{C}-(\omega(X))^{V} U^{C}-(\omega(X))^{C} U^{C}
\end{gathered}
$$

In particular, we have

$$
\begin{gathered}
\tilde{J} X^{V}=-(F X)^{V}, \quad \tilde{J} X^{C}=-(F X)^{C} \\
\tilde{J} U^{V}=U^{C}, \quad \tilde{J} U^{C}=U^{C}
\end{gathered}
$$

$X$ being an arbitrary vector field in $M$ such that $\omega(X)$.

Theorem 2. Let the tangent bundle $T(M)$ of the manifold $M$ admits $\tilde{J}$ defined in (5), then for vector fields $X, Y$ such that $\omega(Y)=0$, we have
(i) $\left(\mathscr{L}_{X^{V}} \tilde{J}\right) Y^{V}=0$,
(ii) $\left.\left(\mathscr{L}_{X^{V}} \tilde{J}\right) Y^{C}=-\left(\left(\mathscr{L}_{X} F\right) Y\right)^{V}+\left(\mathscr{L}_{X} \omega\right) Y\right)^{V} U^{C}$
(iii) $\left(\mathscr{L}_{X^{V}} \widetilde{J}\right) U^{V}=\left(\mathscr{L}_{X} U\right)^{V}$
(iv) $\left.\left(\mathscr{L}_{X^{V}} \tilde{J}\right) U^{C}=-\left(\left(\mathscr{L}_{X} F\right) U\right)^{V}+\left(\mathscr{L}_{X} \omega\right) U\right)^{V} U^{C}$ 
(i) $\left.\left(\mathscr{L}_{X}{ }^{C} \tilde{J}\right) Y^{V}=-\left(\left(\mathscr{L}_{X} F\right) Y\right)^{V}+\left(\mathscr{L}_{X} \omega\right) Y\right)^{V} U^{C}$,

(ii) $\left.\left.\quad\left(\mathscr{L}_{X}{ }^{C} \tilde{J}\right) Y^{C}=-\left(\left(\mathscr{L}_{X} F\right) Y\right)^{C}-\left(\mathscr{L}_{X} \omega\right) Y\right)^{V} U^{C}+\left(\mathscr{L}_{X} \omega\right)\right) Y^{C} U^{C}$

(iii) $\quad\left(\mathscr{L}_{X} \tilde{J}\right) U^{V}=\left(\left(\mathscr{L}_{X} F\right) U\right)^{C}+[X, U]^{C}+\left(\left(\mathscr{L}_{X} \omega\right) U\right)^{V} U^{C}$

(iv) $\quad\left(\mathscr{L}_{X} C \tilde{J}\right) U^{C}=\left(\left(\mathscr{L}_{X} F\right) U\right)^{C}-[X, U]^{C}-\left(\left(\mathscr{L}_{X} \omega\right) U\right)^{V} U^{C}+\left(\left(\mathscr{L}_{X} \omega\right) U\right)^{C} U^{C}$

Proof. The proof follows early from (4), (6), (8), and [3].

\section{Horizontal lifts of Lorentzian almost para contact structure}

Let $(F, U, \omega)$ be Lorentzian almost para contact structure in $\bar{M}$ with an affine connection then (1) and (2) and [3], we have

$$
\begin{array}{r}
\left(F^{H}\right)^{2}=(I+U \otimes \omega)^{H} \\
\left(F^{H}\right)^{2}=I+(U \otimes \omega)^{H} \\
\left(F^{H}\right)^{2}=I+U^{H} \otimes \omega^{V}+U^{V} \otimes \omega^{H}
\end{array}
$$

Also,

$$
\begin{array}{cc}
\text { (i) } & F^{H} U^{H}=0, \quad F^{H} U^{V}=0 \\
\text { (ii) } & \omega^{H}\left(U^{H}\right)=0, \quad \omega^{H}\left(U^{V}\right)=1, \quad \omega^{V}\left(U^{H}\right)=1 \\
\text { (iiI) } & \omega^{H} \circ F^{H}=0, \quad \omega^{V} \circ F^{H}=0 .
\end{array}
$$

Let us define a tenser field $\tilde{J}^{*}$ of type $(1,1)$ in $T(\bar{M})$ by

$$
\tilde{J}^{*}=F^{H}+\left(U^{V} \otimes \omega^{V}-U^{H} \otimes \omega^{H}\right.
$$

then it is easy to show that

$$
\tilde{J}^{*^{2}}=I
$$

thus $\tilde{J}^{*}$ is an almost product structure in $T(\bar{M})$.

Theorem 3. Let $(F, U, \omega)$ be Lorentzian almost para contact structure in $\bar{M}$ with an affine connection $\nabla$. Then $\tilde{J}^{*}$ is almost product structure in $T(\bar{M})$.

\section{Competing interests}

The authors declare that they have no competing interests.

\section{Authors' contributions}

All authors have contributed to all parts of the article. All authors read and approved the final manuscript.

\section{References}

[1] Matsumoto, K. On Lorentzian paracontact manifolds, Bull. of Yamagata Univ. Nat. Sci. Vol. 12, No. 2 (1989), pp. 151-156.

[2] Das, Lovejoy S. and Khan, M. N. I. Almost r - contact structures on the tangent bundle, Differential Geometry -Dynamical Systems, Vol.7 (2005), pp. 34-41. 
[3] Yano, K. and Ishihara, S. Tangent and Cotangent Bundles, Marcel Dekker, Inc. New York, 1973.

[4] Khan, M. N. I. Lifts of hypersurfaces with quarter-symmetric semi-metric connection to tangent bundles, Afrika Matematika Springer-Verlag, Vol.25 No.2 (2014), pp. 475-482.

[5] Blair, D.E. Contact Manifolds in Riemannian Geometry, Lecture Notes in Math, 509, Springer-Verlag, New York, 1976.

[6] Tekkoyun, M. On lifts of paracomplex structures, Turk. J. Math., Vol. 30 (2006), pp. 197-210.

[7] Khan, M. N. I. Lift of semi-symmetric non-metric connection on a Kähler Manifold, Afrika Matematika, Springer Verlag, Vol. 27, No. 3 (2016), pp. 345-352.

[8] Yano, K. and Ishihara, S. Almost complex structures induced in tangent bundles. Kodai Math. Sem. Rep., 1967, 19, 1-27.

[9] Davies, E.T. On the curvature of the tangent bundle, Annali di Mat. 1969, (IV) 81, 193-204.

[10] Yano, K.and Davies, E.T. Metrics and connections in tangent bundle, Kodai Math. Sem. Rep., 1971, 23, 493-504. 